\title{
ATMOSPHERIC DISPERSION MODELING OF BASIS-DESIGN ACCIDENT (LOCA) AND RADIOACTIVE DOSE ASSESSMENT OF TWO PROPOSED SITES FOR NUCLEAR POWER PLANT IN TUNISIA
}

\author{
Riadh Souissi \\ Department of Nuclear Safety \\ National Center for Nuclear sciences and Technologies, \\ Tunis, Tunisia \\ Abou Bakr Ramadan \\ Department of Siting and Environment \\ Nuclear and Radiological Regulatory Authority, \\ Cairo, Egypt
}

Abstract - The demand for energy is constantly increasing and nuclear power remains an important option for many countries to provide energy for development and fight climate change. In Tunisia, two sites are preselected for Nuclear Power Plant (NPP) implantation: Marsa-Dhouiba site in the far north of the country and Skhira site in the South-East. Estimating of health effects on humans (population and workers) following the accidental release of radioactive gaseous materials into the air from a Nuclear Power Plant is essential for the safety and environmental analyses that are needed for reactor licensing. So, the early offsite consequences of severe accident should be assessed before construction of proposed NPP for many purposes, such as site evaluation of NPP, evaluation of the exclusion area and low population zone. Also, the emergency plan must be evaluated to define the mitigation actions. These actions may represented by sheltering, evacuation, distribution of stable iodine tablets and/or relocation and where these actions should take place after the nuclear accident especially in the downwind sectors to decrease the consequences of accident. The hypothetical scenario considered in this paper is a design-basis accident, loss of coolant accident. The RASCAL code was applied to evaluate the health effects and define the protective actions in the event of a nuclear accident for both sites. The health effects can be treated as two important dosimetric parameters: the total effective dose equivalent (TEDE) around the reactor sites and the thyroid commitment dose equivalent (TCDE). After making an analysis of meteorological parameters for both sites, atmospheric

\author{
Faten Tawfik \\ Department of Siting and Environment \\ Nuclear and Radiological Regulatory Authority, \\ Cairo, Egypt \\ Nafaa Reguigui \\ Department of Nuclear Safety \\ National Center for Nuclear sciences and Technologies, \\ Tunis, Tunisia
}

dispersion modeling is made for each of the four seasons and finally recommendations for immediate protective actions are discussed.

Keywords - Nuclear accidents, Evacuation, Sheltering, Nuclear power plants, Protective action recommendations, LOCA

\section{INTRODUCTION}

In Tunisia, two sites, shown in Figure 1, are preselected for Nuclear Power Plant (NPP) implantation: Marsa-Dhouiba site in the far north of the country (N3716, E0929) and Skhira site in the South-East (N3415, E1003) in Gabes Gulf. The meteorological data of both sites are taken from stations established in-situ at an altitude of $24 \mathrm{~m}$ above sea level and recorded different meteorological conditions.

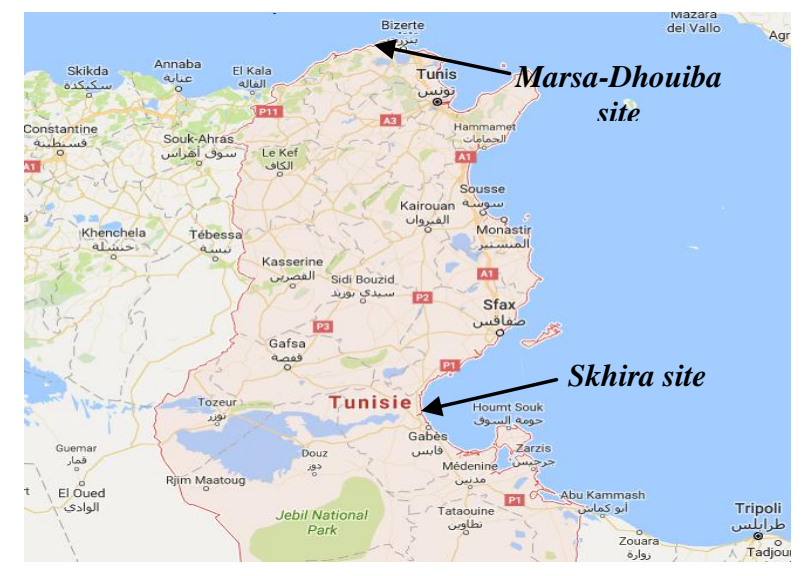


Fig. 1. Geographic localisation of the two preselected tunisian sites

The Marsa Dhouiba site, located in Kef Abbed region (Bizerte government), is connected to the Mediterranean Sea. The land is vegetative, with a loamy soil. Its climate is mild, damp and temperature average is $22.8{ }^{\circ} \mathrm{C}$. Kef Abbed is a small isolated village on sea frequented by some local fishermen rarely visited and characterized by small population dispersed not more than 1000. The capital Tunis is located about $60 \mathrm{Km}$ from this site in a straight line in the South-East direction.

The Skhira site (Sfax government), located in Gabes gulf, and is also connected to the Mediterranean Sea. The study area is subject to two opposite types of climate action: climate Saharan dry and hot in the southwest and the Mediterranean climate, relatively wet. According to the Tunisian's census of 2014 , the Skhira site is a municipality of 34,673 habitants in 2014. The capital Tunis is located about $280 \mathrm{Km}$ from this site in a straight line in the North direction. Table 1 below describes the meteorological stations and the environments of both sites.

Table 1. Meteorological stations position and environments

\begin{tabular}{|c|c|c|c|c|c|c|c|}
\hline Site & $\begin{array}{c}\text { Lat } \\
\left({ }^{\circ},{ }^{\prime}\right)\end{array}$ & $\begin{array}{c}\text { Long } \\
\left({ }^{\circ},{ }^{\prime}\right)\end{array}$ & Position & $\begin{array}{c}\text { Distance } \\
\text { km } \\
\text { wind } \\
\text { direction }\end{array}$ & Soil & Vegetation & Aridity \\
\hline $\begin{array}{c}\text { Marsa } \\
\text { Dhouiba }\end{array}$ & 3716 & 0929 & Coast & $\begin{array}{c}0.450 \\
\mathrm{~km} \text { NO }\end{array}$ & light & $\begin{array}{c}\text { Fallow + } \\
\text { forest }\end{array}$ & humid \\
\hline Skhira & 3415 & 1003 & $\begin{array}{c}\text { Large } \\
\text { lowland }\end{array}$ & $\begin{array}{c}1.2 \\
\mathrm{Km} / \mathrm{E}\end{array}$ & medium & $\begin{array}{c}\text { Fallow + } \\
\text { olive }\end{array}$ & arid \\
\hline
\end{tabular}

In the present work, an accidental release scenario will be considered and simulated using RASCAL code. The software use Gaussian Plume Models (GPM) to describe the atmospheric dispersion of radioactive material. In general, GPM is widely used in literature and has been successfully applied to many dispersion problems (Bamdad and Hosseini, 2016; Denglong and Zaoxiao, 2016; Benamrane and Boustras, 2015; Pirouzmand et al. 2015; Timothy, 2013).

Hypothetical accidents release radionuclides are based upon literature review and particularly on NRC's technical reports [US NRC 10 C.F.R. Part 52, "Early Site Permits; Standard Design Certifications; and Combined Licenses for Nuclear Power Plants,", a typical design-basis Loss Of Coolant Accident (LOCA) is simulated using RASCAL code.

The accidental scenario assumes that the core is maintained uncovered for a period of time $(120 \mathrm{mn})$ leading a significant released activity. The accidental atmospheric dispersion will be modeled by RASCAL software. The results concern primarily two major dosimetric parameters: the Total Effective Dose Equivalent (TEDE) and the Thyroid Commitment Dose Equivalent (TCDE). TEDE and TCDE will be compared to dose's threshold to be able to undertake the appropriate protections actions recommended by the U.S. Environmental Protection Agency (U.S. EPA, 2013).

\section{ATMOSPHERIC DISPERSION MODELING FOLLOWING AN ACCIDENT}

We focus on studying atmospheric dispersion following a postulated loss of coolant accident (LOCA) and we assume that nuclear plant's emergency core cooling system (ECCS) no longer exists. The LOCA could be also defined as an uncovered core events accident. This specific accident will be treated with RASCAL software.

In the following sections, we will introduce two main results obtained from RASCAL which are: the Total Effective Dose Equivalent (TEDE) including all possible doses for external and internal exposures received and the Thyroid Committed Dose Equivalent (TCDE). TEDE and TCDE will be, therefore, compared to EPA's limits. In fact during an incident with an uncontrolled source of radiation, protection of the public from unnecessary exposure to radiation may require some form of intervention that will disrupt normal living. Such intervention is termed a protective action (U.S. EPA, 2013). Among protective actions: evacuating an area; sheltering-in-place within a building or protective structure; administering potassium iodide (KI) as a supplemental action; acquiring an alternate source of drinking water; interdiction of food $/ \mathrm{milk}$. The following table lists the thresholds for each of these actions (U.S. EPA, 2013).

Table -2 Recommended protective actions for the Early Phase of a Radiological Incident

\begin{tabular}{|c|c|c|}
\hline Actions & Dose thresholds [mSv] & Comments \\
\hline Sheltering & 10 & $\begin{array}{c}\text { The total dose by external } \\
\text { exposure and the } \\
\text { committed effective dose } \\
\text { for internal exposure } \\
\text { during 4 days }\end{array}$ \\
\hline $\begin{array}{c}\text { Potassium Iodine } \\
\text { (KI) admission }\end{array}$ & $\begin{array}{c}50 \text { received on thyroid } \\
\text { child by exposure to } \\
\text { Iodine }\end{array}$ & $\begin{array}{c}\text { After the approval of a } \\
\text { medical team }\end{array}$ \\
\hline
\end{tabular}

The Food and Drug Administration (FDA) updated its guidance on the use of KI as a thyroid blocking agent during radiological emergencies in 2001 (U.S.FDA, 2001, 2002). FDA based these dose recommendations on a review of the thyroid cancer data from the Chernobyl reactor accident of April 1986 and the experience of Poland in administering KI following the Chernobyl release (FDA, 2001). FDA recommends the following:

Children 0-18 years of age: Administer KI when the projected radiation dose to the thyroid from exposure to radioiodine is $5 \mathrm{rem}(50 \mathrm{mSv})$ or greater.

Pregnant and lactating women: Administer KI when the projected radiation dose to the thyroid from exposure to radioiodine is $5 \mathrm{rem}(50 \mathrm{mSv})$ or greater. 
Adults up to 40 years of age: Administer KI when the projected radiation dose to the thyroid from exposure to radioiodine is $10 \mathrm{rem}(100 \mathrm{mSv})$ or greater.

Adults over 40 years of age: Administer KI when the projected radiation dose to the thyroid from exposure to radioiodine is over $500 \mathrm{rem}$ in order to prevent hypothyroidism.

\section{II.1. RASCAL description}

RASCAL (Radiological Assessment System for Consequence Analysis) is currently used by NRC's emergency operations center for making dose projections for atmospheric releases during radiological emergencies (McGuirea et al. 2007). The code is widely used in several recent scientific work of radiological assessment (Bo et al. 2016; Rossi et al. 2014; Benamrane et al. 2013; Muswema et al. 2014, Schmidt, and Ivanov 2012). RASCAL 3.0.5 uses Gaussian models to describe the atmospheric dispersion of radioactive and chemical effluents from nuclear facilities. In the calculation, RASCAL flip between two models: (i) the straight-line Gaussian plume model (Equation 1) used near the release point where travel times are short and plume depletion associated with dry deposition is small and (ii) the Lagrangian-trajectory Gaussian puff model (Equation 2) used at longer distances where temporal or spatial variations in meteorological conditions and depletion of the plume due to dry deposition may be significant.

RASCAL was chosen to model the accidental atmospheric dispersion, and to compare between the two sites of nuclear safety point of view and finally recommend possible convenient radiological emergencies such as define evacuation and sheltering zones.

\section{II.1.1 Straight-Line Gaussian Plume Models}

Puff models represent plumes as a series of puffs. Concentrations at a point in the plume are calculated by adding the concentrations at the point associated with all puffs in the vicinity of the point. The simplified version of the straight-line Gaussian model used in RASCAL is given by:

$$
\mathrm{X}(x, y, z) / \mathrm{Q}^{\prime}=\mathrm{FyFz} / 2 \pi \mathrm{u} \sigma_{\mathrm{y}} \sigma_{\mathrm{z}}
$$

where

$\mathrm{X}=$ average concentration, $\mathrm{Q}^{\prime}=$ release rate, $\mathrm{FyFz}=$ lateral and vertical exponential terms, $x=$ downwind distance at which $\mathrm{X}, \sigma_{\mathrm{x}}, \sigma_{\mathrm{y}}$ and $\sigma_{\mathrm{z}}$ are evaluated, $\mathrm{u}=$ wind speed and $\mathrm{t}=$ time.

\section{II.1.2 Gaussian Puff Model}

In a Cartesian coordinate system with $\mathrm{x}$ and $\mathrm{y}$ axes in a horizontal plane and $\mathrm{z}$ in the vertical, the normalized concentration in the vicinity of the puff are:

$$
\begin{gathered}
\mathrm{X}(x, y, z) / \mathrm{Q}=\left(1 /(2 \pi)^{3 / 2} \sigma_{\mathrm{x}} \sigma_{\mathrm{y}} \sigma_{\mathrm{z}}\right) \exp \left[-0.5\left(\left(x-x_{0}\right) / \sigma_{\mathrm{x}}\right)^{2}\right] \exp [- \\
\left.0.5\left(\left(y-y_{0}\right) / \sigma_{\mathrm{y}}\right)^{2}\right] \exp \left[-0.5\left(\left(z-z_{0}\right) / \sigma_{z}\right)^{2}\right]
\end{gathered}
$$

\section{II.2. Description of Inputs and Scenario of release}

First, we define the location of the Nuclear Power Plant based on latitude and longitude of Table 1. Based on maximum allowed capacity of electrical network, Tunisian electrical utility (STEG) recommends a pressurized water reactor with an effective electric power which should not exceed 1000 MWe. Based on these recommendations we have considered that following reactor parameters:

Table -3 Reactor parameters introduced in RASCAL calculations

\begin{tabular}{|l|c|}
\hline Reactor power & 3300 MW(t) \\
\hline Average fuel burn-up & $22000 \mathrm{MWD} / \mathrm{MTU}$ \\
\hline Containment type & PWR Dry Ambient \\
\hline Containment volume & $2.50 \mathrm{E}+06 \mathrm{ft}^{3}$ \\
\hline Assemblies in core & 200 \\
\hline Stream generator type & U-Tube \\
\hline
\end{tabular}

\section{II.2.1 Straight-Line Gaussian Plume Models}

If we specify how long the core is uncovered, RASCAL will estimate how much core damage will occur. Table 4 was taken from Table 3.13 in NUREG-1465 (Soffer et al. 1995.) For example, if a PWR core is uncovered for 15 or 30 minutes, the estimated damage is $50 \%$ or $100 \%$ cladding failure, respectively.

Table 4. PWR Event Timings and Fraction of Core Activity Inventory Released

\begin{tabular}{|l|c|c|c|}
\hline \multirow{2}{*}{ Nuclide group } & \multicolumn{3}{|c|}{ PWR core inventory release fraction* } \\
\cline { 2 - 4 } & $\begin{array}{l}\text { Cladding } \\
\text { failure } \\
\text { (gap release } \\
\text { phase) } \\
\text { (0.5 hr) }\end{array}$ & $\begin{array}{l}\text { Core melt } \\
\text { phase } \\
\text { (in-vessel } \\
\text { phase) } \\
\text { (1.3 hr) }\end{array}$ & $\begin{array}{l}\text { Post-vessel } \\
\text { melt-through } \\
\text { phase (ex- } \\
\text { vessel phase) } \\
\text { (2.0 hr) }\end{array}$ \\
\hline Noble gases (Kr, Xe) & 0.05 & 0.95 & 0 \\
\hline Halogens (I, Br) & 0.05 & 0.35 & 0.25 \\
\hline Alkali metals (Cs, Rb) & 0.05 & 0.25 & 0.35 \\
\hline $\begin{array}{l}\text { Tellerium group (Te, Sb, } \\
\text { Se) }\end{array}$ & 0 & 0.05 & 0.25 \\
\hline $\begin{array}{l}\text { Barium, strontium (Ba, } \\
\text { Sr) }\end{array}$ & 0 & 0.02 & 0.1 \\
\hline $\begin{array}{l}\text { Noble metals (Ru, Rh, } \\
\text { Pd, Mo, Tc, Co) }\end{array}$ & 0 & 0.0025 & 0.0025 \\
\hline $\begin{array}{l}\text { Cerium group (Ce, Pu, } \\
\text { Np) }\end{array}$ & 0 & 0.0005 & 0.005 \\
\hline $\begin{array}{l}\text { Lanthanides (La, Zr, Nd, } \\
\text { Eu, Nb, Pm, Pr, Sm, Y, } \\
\text { Cm, Am) }\end{array}$ & 0 & 0.0002 & 0.005 \\
\hline
\end{tabular}

*Reference: (Table 3.13 de NUREG-1465 (Soffer et al. 1995)

To assess the source terms, we choose "time core is uncovered" option in RASCAL to quantify it. A $30 \mathrm{~m}$ height 
containment bypass is used as a release pathway in the calculations. 30 meters is approximately the containment height At the beginning of the accident, a reactor shutdown occurs and simultaneously the core become totally uncovered by loss of coolant and remains uncovered for two hours (120 minutes) which corresponds to a post-vessel melt through phase as mentioned above in third column of Table 4.

\section{II.2.2 Models Meteorological data introduced}

Taking into account the analysis of meteorological parameters for both sites, we consider the following meteorological data in our simulations as indicated in Table 5.

Table 5. Activity Inputs used in RASCAL simulations

\begin{tabular}{|c|c|c|c|c|c|}
\hline Site & Season & $\begin{array}{l}\text { Prevailing } \\
\text { Direction }\end{array}$ & $\begin{array}{l}\text { Wind } \\
\text { speed } \\
{[\mathrm{m} / \mathrm{s}]}\end{array}$ & $\begin{array}{c}\text { Relative } \\
\text { Humidity } \\
{[\%]}\end{array}$ & $\begin{array}{c}\text { Stability } \\
\text { class }\end{array}$ \\
\hline \multirow{4}{*}{ 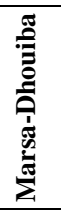 } & Autumn & $120^{\circ}$ & 6 & 70 & D \\
\hline & Winter & $270^{\circ}$ & 6 & 80 & D \\
\hline & Springer & $270^{\circ}$ & 6 & 75 & $\mathrm{D}$ \\
\hline & Summer & $270^{\circ}$ & 7 & 60 & B \\
\hline \multirow{4}{*}{$\frac{\pi}{\pi}$} & Autumn & $360^{\circ}$ & 6 & 65 & $\mathrm{C}$ \\
\hline & Winter & $250^{\circ}$ & 8 & 60 & $\mathrm{C}$ \\
\hline & Springer & $90^{\circ}$ & 8 & 60 & $\mathrm{D}$ \\
\hline & Summer & $90^{\circ}$ & 8 & 60 & $\mathrm{C}$ \\
\hline
\end{tabular}

\section{RESULTS AND DISCUSSION}

Results are given for each of four seasons where a day was chosen from each season to conduct simulation and in which the accident occurs represents well the meteorological data averaged over the season. Finally the simulation was carried for worst release. Cumulative TEDE and TCDE are given for 30 hours after the beginning of the release which is a sufficient period to undertake protective actions.

\section{III.1. Atmospheric dispersion during winter \\ III.1.1 TEDE}

During winter, the TEDE obtained in Marsa-Dhouiba site is higher than that of Skhira site (Figure 2 and Table 6). This is reflected by the impact area of dose exceeding $50 \mathrm{mSv}$ and 10 $\mathrm{mSv}$ for Marsa-Dhouiba and Skhira, respectively. Whereas, the impact area of TEDE exceeding $0.01 \mathrm{mSv}$ is three times higher in Skhira site than in Marsa Dhouiba site but this finding is not so important because all the green area doesn't represent any danger. Table 6 gives the main numerical results. (a)

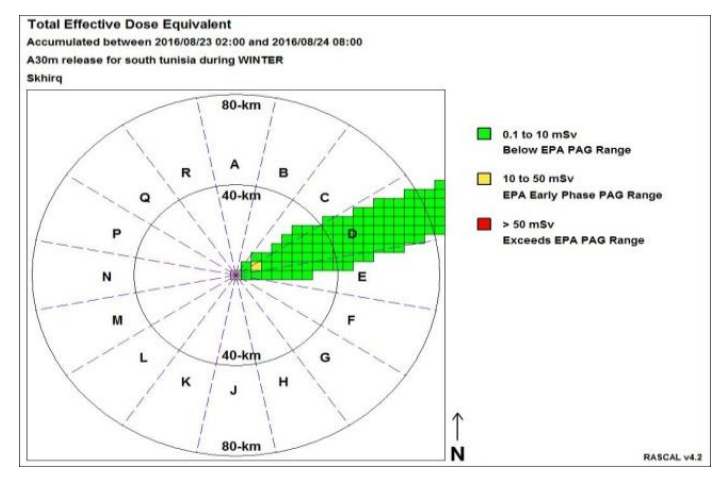

(b)

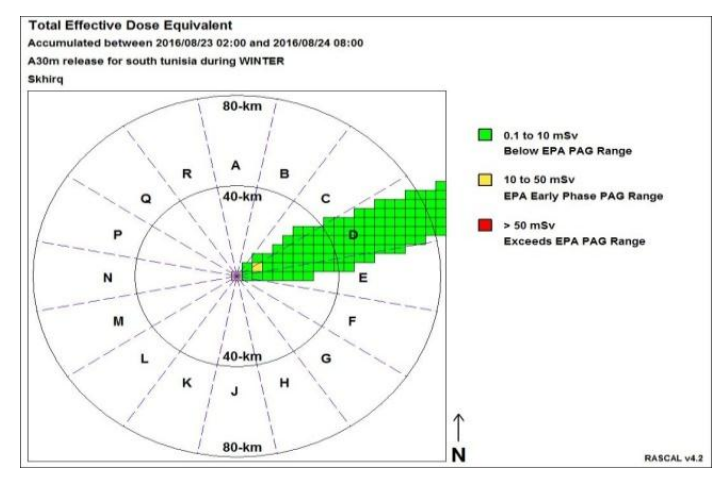

Fig. 2. Schematization of Simulated TEDE obtained during winter season (a) Marsa-Dhouiba site (Met. Stab class, D) (b) Skhira site (Met. Stab class, C)

Table 6. RASCAL's TEDE outputs obtained during winter for both

\begin{tabular}{|c|c|c|c|c|c|}
\hline \multicolumn{6}{|c|}{ sites } \\
\hline Site & $\begin{array}{c}\text { Maximum } \\
\text { dose [Sv] } \\
\text { (Distance } \\
[\mathrm{m}])\end{array}$ & $\begin{array}{c}\text { Threshold } \\
\text { TEDE > }\end{array}$ & $\begin{array}{c}\text { Impact } \\
\text { area } \\
{[\mathrm{km} 2]}\end{array}$ & $\begin{array}{c}\text { Maximum } \\
\text { distance } \\
\text { reached } \\
{[\mathbf{k m}]} \\
\text { (values } \\
[\mathrm{mSv}])\end{array}$ & $\begin{array}{l}\text { Recommended } \\
\text { immediate } \\
\text { protective } \\
\text { actions }\end{array}$ \\
\hline \multirow{3}{*}{ 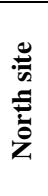 } & \multirow{3}{*}{$\begin{array}{c}2.36 \\
(800)\end{array}$} & $50 \mathrm{mSv}$ & 48.6 & $\begin{array}{l}11.27 \\
(70.1)\end{array}$ & $\begin{array}{c}\text { Evacuation to } \\
11.27 \mathrm{~km}\end{array}$ \\
\hline & & $10 \mathrm{mSv}$ & 97.1 & $\begin{array}{c}20.0 \\
(11.2) \\
\end{array}$ & $\begin{array}{c}\text { Sheltering to } \\
20.0 \mathrm{~km}\end{array}$ \\
\hline & & $0.01 \mathrm{mSv}$ & 987.4 & $\begin{array}{c}80 \\
(1.39)\end{array}$ & $\begin{array}{c}\text { Safe area from } \\
20 \mathrm{~km}\end{array}$ \\
\hline \multirow{3}{*}{ 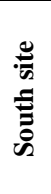 } & \multirow{3}{*}{$\begin{array}{c}8.6110-01 \\
(800)\end{array}$} & $50 \mathrm{mSv}$ & 0.7 & $\begin{array}{c}8.0 \\
(50.2)\end{array}$ & $\begin{array}{c}\text { Evacuation to } \\
8.0 \mathrm{~km}\end{array}$ \\
\hline & & $10 \mathrm{mSv}$ & 32.4 & $\begin{array}{c}16 \\
(16.3)\end{array}$ & $\begin{array}{c}\text { Sheltering to } 16 \\
\mathrm{~km}\end{array}$ \\
\hline & & $0.01 \mathrm{mSv}$ & 2379.6 & $\begin{array}{c}80 \\
(0.4)\end{array}$ & $\begin{array}{c}\text { Safe area from } \\
16 \mathrm{~km}\end{array}$ \\
\hline
\end{tabular}

\section{III.1.2 Thyroid commitment dose equivalent}

The spread of the TCDE is similar to that of TEDE but with smaller impacted areas. Wherever, we notice in Figure 3.a the presence of a yellow zone which corresponds to areas within which TDC is included between 50 and $250 \mathrm{mSv}$ while for Skhira site there are only safety areas (green zone). Table 7 gives the outputs obtained during winter for both sites. 
(a)

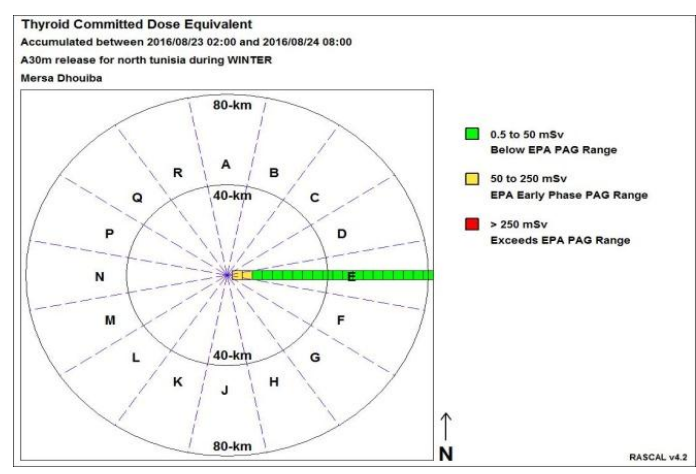

(b)

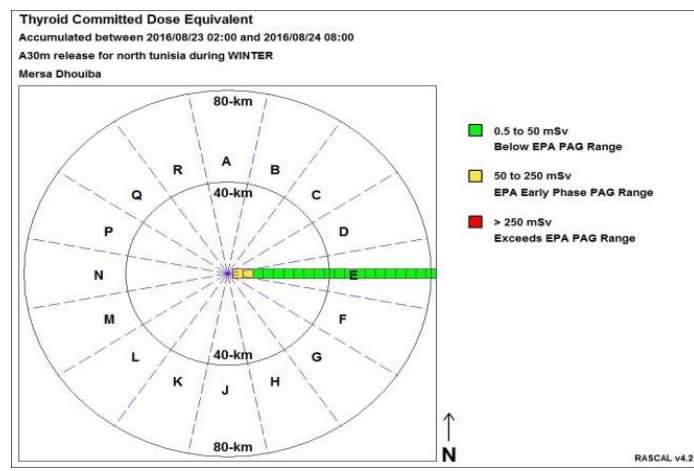

Fig. 3. Schematization Thyroid Commitment Dose obtained during winter season (a) Marsa-Dhouiba site (Met. Stab class, D) (b) Skhira site (Met. Stab class, C)

Table 7. RASCAL's TCDE outputs obtained during winter for both

\begin{tabular}{|c|c|c|c|c|c|}
\hline Site & $\begin{array}{c}\text { Maximum } \\
\text { dose [Sv] } \\
\text { (Distance } \\
[\mathrm{m}])\end{array}$ & $\begin{array}{c}\text { Threshold } \\
\text { TCDE > }\end{array}$ & $\begin{array}{c}\text { Impact } \\
\text { area } \\
{[\mathrm{km} 2]}\end{array}$ & $\begin{array}{c}\text { Maximum } \\
\text { distance } \\
\text { reached } \\
{[\mathrm{km}]} \\
\text { (values } \\
[\mathrm{mSv}])\end{array}$ & $\begin{array}{l}\text { Recommended } \\
\text { immediate } \\
\text { protective actions }\end{array}$ \\
\hline \multirow{3}{*}{ 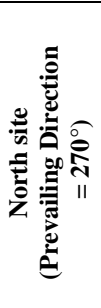 } & \multirow{3}{*}{$\begin{array}{l}5.35 \\
(800)\end{array}$} & $250 \mathrm{mSv}$ & 0.20 & $\begin{array}{c}3.22 \\
(83.9)\end{array}$ & $\begin{array}{l}\mathrm{KI} \text { admission for } \\
\text { adults up to } 40 \text { years } \\
\text { for under } 3.22 \mathrm{~km}\end{array}$ \\
\hline & & $50 \mathrm{mSv}$ & 32.4 & $\begin{array}{c}8.40 \\
(55.6)\end{array}$ & $\begin{array}{c}\text { KI admission for } \\
\text { children, pregnant } \\
\text { and lactating woman } \\
\text { for under } 8.4 \mathrm{~km} \\
\end{array}$ \\
\hline & & $0.05 \mathrm{mSv}$ & 809.4 & $\begin{array}{l}80.00 \\
(0.52)\end{array}$ & $\begin{array}{c}\text { Safe area from } 8.4 \\
\mathrm{~km}\end{array}$ \\
\hline \multirow{3}{*}{ 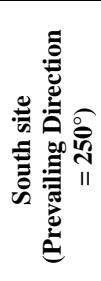 } & \multirow{3}{*}{$\begin{array}{c}1.37 \\
(800)\end{array}$} & $250 \mathrm{mSv}$ & 0.00 & $\begin{array}{c}1.61 \\
(346.00)\end{array}$ & $\begin{array}{l}\mathrm{KI} \text { admission for } \\
\text { adults up to } 40 \text { years } \\
\text { for under } 1.61 \mathrm{~km}\end{array}$ \\
\hline & & $50 \mathrm{mSv}$ & 0.20 & $\begin{array}{c}3.22 \\
(83.90)\end{array}$ & Sheltering to $16 \mathrm{~km}$ \\
\hline & & $0.05 \mathrm{mSv}$ & 1861.6 & $\begin{array}{l}74.00 \\
(0.39)\end{array}$ & $\begin{array}{l}\text { Safe area from } 16 \\
\mathrm{~km}\end{array}$ \\
\hline
\end{tabular}

\section{III.2. Atmospheric dispersion during autumn}

III.2.1 TEDE
During autumn season, the TEDE obtained in Skhira site is higher than that of Marsa-Dhouiba site (Figure 4 and Table 8). This is reflected by the impact area of dose exceeding respectively $50 \mathrm{mSv}, 10 \mathrm{mSv}$ and $0.01 \mathrm{mSv}$.

(a)

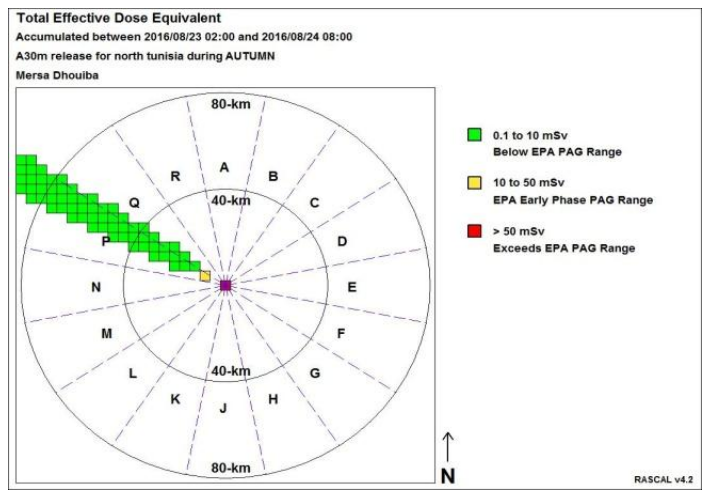

(b)

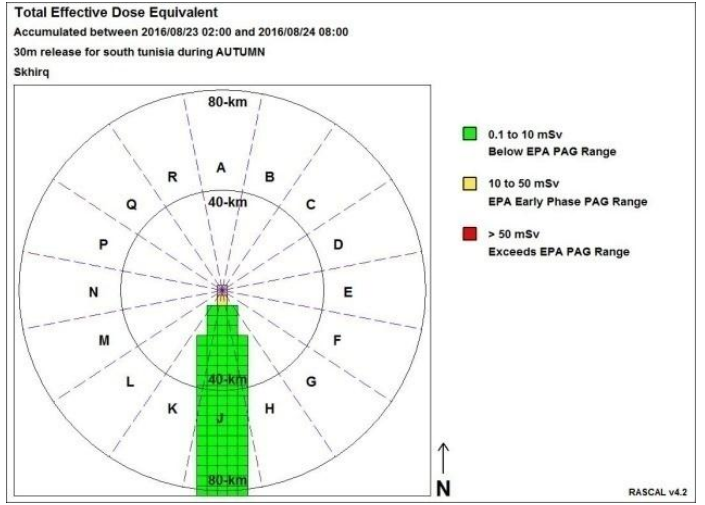

Fig. 4. Schematization of Simulated TEDE obtained during autumn season (a) Marsa-Dhouiba site (Met. Stab class, D) (b) Skhira site (Met. Stab class, C)

Table 8. RASCAL's TEDE outputs obtained during autumn for both sites

\begin{tabular}{|c|c|c|c|c|c|}
\hline Site & $\begin{array}{c}\text { Maximum } \\
\text { dose }[\mathrm{Sv}] \\
\text { (Distance } \\
[\mathrm{m}])\end{array}$ & $\begin{array}{l}\text { Threshold } \\
\text { TEDE > }\end{array}$ & $\begin{array}{c}\text { Impact } \\
\text { area } \\
{[\mathrm{km} 2]}\end{array}$ & $\begin{array}{c}\text { Maximum } \\
\text { distance } \\
\text { reached } \\
{[\mathrm{km}]} \\
\text { (values } \\
\text { [mSv]) } \\
\end{array}$ & $\begin{array}{l}\text { Recommended } \\
\text { immediate } \\
\text { protective } \\
\text { actions }\end{array}$ \\
\hline \multirow{3}{*}{ 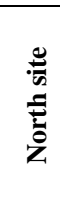 } & \multirow{3}{*}{$\begin{array}{l}1.18 \\
(800)\end{array}$} & $50 \mathrm{mSv}$ & 16.2 & $\begin{array}{c}4.83 \\
(71.2)\end{array}$ & $\begin{array}{c}\text { Evacuation to } \\
4.83 \mathrm{~km}\end{array}$ \\
\hline & & $10 \mathrm{mSv}$ & 32.4 & $\begin{array}{c}8.0 \\
(17.6) \\
\end{array}$ & $\begin{array}{c}\text { Sheltering to } \\
8.0 \mathrm{~km}\end{array}$ \\
\hline & & $0.01 \mathrm{mSv}$ & 1262.1 & $\begin{array}{c}80 \\
(0.52)\end{array}$ & $\begin{array}{c}\text { Safe area from } \\
8 \mathrm{~km}\end{array}$ \\
\hline \multirow{3}{*}{ 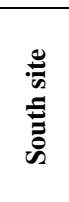 } & \multirow{3}{*}{$\begin{array}{l}0.496 \\
(800)\end{array}$} & $50 \mathrm{mSv}$ & 0.00 & $\begin{array}{c}2.41 \\
(77.0)\end{array}$ & $\begin{array}{c}\text { Evacuation to } \\
2.41 \mathrm{~km}\end{array}$ \\
\hline & & $10 \mathrm{mSv}$ & 32.4 & $\begin{array}{c}4.48 \\
(15.2)\end{array}$ & $\begin{array}{c}\text { Sheltering to } \\
4.48 \mathrm{~km}\end{array}$ \\
\hline & & $0.01 \mathrm{mSv}$ & 1958.7 & $\begin{array}{c}80 \\
(1.04)\end{array}$ & $\begin{array}{c}\text { Safe area from } \\
4.48 \mathrm{~km}\end{array}$ \\
\hline
\end{tabular}


III.2.2 Thyroid Commitment Dose

(a)

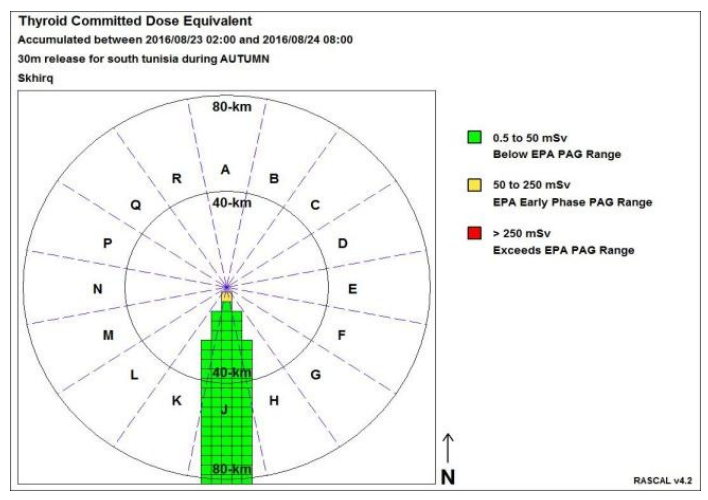

(b)

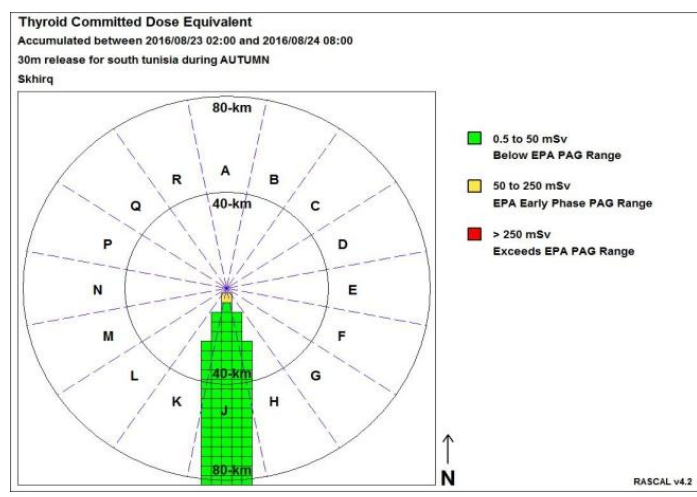

Fig. 5. Schematization Thyroid Commitment Dose obtained during autumn season (a) Marsa-Dhouiba site (Met. Stab class, D) (b) Skhira site (Met. Stab class, C)

Table 9. RASCAL's TCDE outputs obtained during autumn for both

\begin{tabular}{|c|c|c|c|c|c|}
\hline \multicolumn{6}{|c|}{ sites } \\
\hline Site & $\begin{array}{c}\text { Maximum } \\
\text { dose [Sv] } \\
\text { (Distance } \\
[\mathbf{m}])\end{array}$ & $\begin{array}{c}\text { Threshold } \\
\text { TCDE > }\end{array}$ & $\begin{array}{l}\text { Impact } \\
\text { area } \\
{[\mathrm{km} 2]}\end{array}$ & $\begin{array}{c}\text { Maximum } \\
\text { distance } \\
\text { reached } \\
{[\mathrm{km}]} \\
\text { (values } \\
[\mathrm{mSv}])\end{array}$ & $\begin{array}{l}\text { Recommended } \\
\text { immediate } \\
\text { protective actions }\end{array}$ \\
\hline \multirow{3}{*}{ 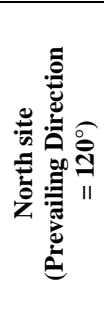 } & \multirow{3}{*}{$\begin{array}{l}5.51 \\
(800)\end{array}$} & $250 \mathrm{mSv}$ & 0.10 & $\begin{array}{c}3.22 \\
(419.00)\end{array}$ & $\begin{array}{l}\text { KI admission for } \\
\text { adults up to } 40 \text { years } \\
\text { for under } 3.20 \mathrm{~km}\end{array}$ \\
\hline & & $50 \mathrm{mSv}$ & 0.40 & $\begin{array}{c}8.04 \\
(74.50)\end{array}$ & $\begin{array}{c}\text { KI admission for } \\
\text { children, pregnant } \\
\text { and lactating woman } \\
\text { for under } 8.04 \mathrm{~km}\end{array}$ \\
\hline & & $0.05 \mathrm{mSv}$ & 1052.2 & $\begin{array}{l}80.00 \\
(1.83)\end{array}$ & $\begin{array}{l}\text { Safe area from } 8.4 \\
\qquad \mathrm{~km}\end{array}$ \\
\hline \multirow{3}{*}{ 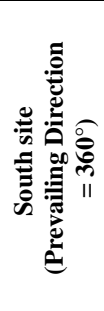 } & \multirow{3}{*}{$\begin{array}{c}1.78 \\
(800)\end{array}$} & $250 \mathrm{mSv}$ & 0.00 & $\begin{array}{c}1.61 \\
(468.00)\end{array}$ & $\begin{array}{l}\text { KI admission for } \\
\text { adults up to } 40 \text { years } \\
\text { for under } 1.61 \mathrm{~km}\end{array}$ \\
\hline & & $50 \mathrm{mSv}$ & 0.30 & $\begin{array}{c}8.40 \\
(53.30)\end{array}$ & $\begin{array}{c}\text { KI admission for } \\
\text { children, pregnant } \\
\text { and lactating woman } \\
\text { for under } 8.40 \mathrm{~km}\end{array}$ \\
\hline & & $0.05 \mathrm{mSv}$ & 1910.4 & $\begin{array}{l}80.00 \\
(3.55)\end{array}$ & $\begin{array}{c}\text { Safe area from } \\
8.4 \mathrm{~km}\end{array}$ \\
\hline
\end{tabular}

\section{III.3. Atmospheric dispersion during springer}

\section{III.3.1 TEDE}

(a)

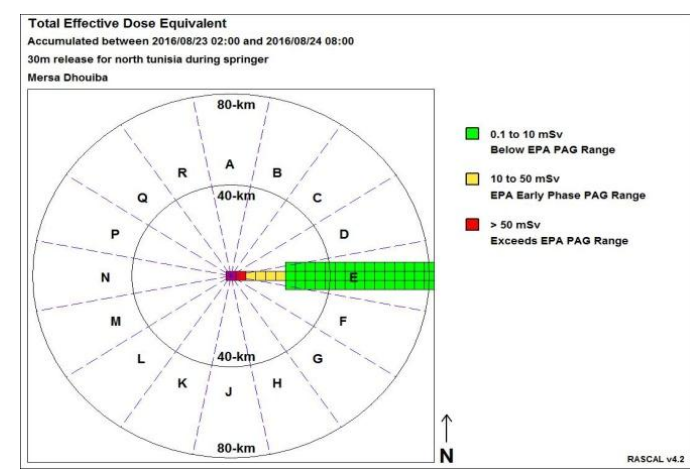

(b)

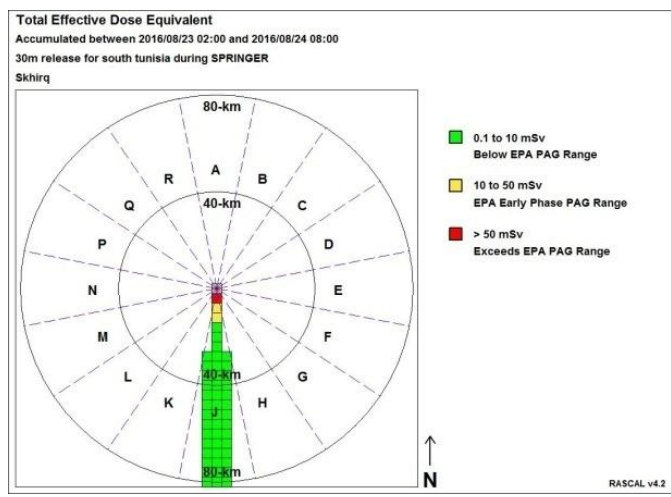

Fig. 6. Schematization of Simulated TEDE obtained during springer season (a) Marsa-Dhouiba site (Met. Stab class, D) (b) Skhira site (Met. Stab class, C)

Table 10. RASCAL's TEDE outputs obtained during springer for

\begin{tabular}{|c|c|c|c|c|c|}
\hline \multicolumn{6}{|c|}{ both sites } \\
\hline Site & $\begin{array}{c}\text { Maximum } \\
\text { dose }[\mathrm{Sv}] \\
\text { (Distance } \\
[\mathrm{m}])\end{array}$ & $\begin{array}{c}\text { Threshold } \\
\text { TEDE > }\end{array}$ & $\begin{array}{c}\text { Impact } \\
\text { area } \\
{[\mathrm{km} 2]}\end{array}$ & $\begin{array}{c}\text { Maximum } \\
\text { distance } \\
\text { reached } \\
{[\mathrm{km}]} \\
\text { (values } \\
[\mathrm{mSv}])\end{array}$ & $\begin{array}{l}\text { Recommended } \\
\text { immediate } \\
\text { protective } \\
\text { actions }\end{array}$ \\
\hline \multirow{3}{*}{ 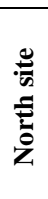 } & \multirow{3}{*}{$\begin{array}{c}1.19 \\
(800)\end{array}$} & $50 \mathrm{mSv}$ & 0.20 & $\begin{array}{c}4.80 \\
(71.80)\end{array}$ & $\begin{array}{c}\text { Evacuation to } \\
4.8 \mathrm{~km}\end{array}$ \\
\hline & & $10 \mathrm{mSv}$ & 1.30 & $\begin{array}{c}20.00 \\
(10.60)\end{array}$ & $\begin{array}{l}\text { Sheltering to } 20 \\
\mathrm{~km}\end{array}$ \\
\hline & & $0.01 \mathrm{mSv}$ & 4.10 & $\begin{array}{l}80.00 \\
(2.74)\end{array}$ & $\begin{array}{l}\text { Safe area from } \\
20 \mathrm{~km}\end{array}$ \\
\hline \multirow{3}{*}{ 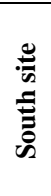 } & \multirow{3}{*}{$\begin{array}{c}1.02 \\
(800)\end{array}$} & $50 \mathrm{mSv}$ & 0.20 & $\begin{array}{c}4.83 \\
(59.2)\end{array}$ & $\begin{array}{c}\text { Evacuation to } \\
4.83 \mathrm{~km}\end{array}$ \\
\hline & & $10 \mathrm{mSv}$ & 0.40 & $\begin{array}{c}8.00 \\
(17.2) \\
\end{array}$ & $\begin{array}{l}\text { Sheltering to } 8 \\
\mathrm{~km}\end{array}$ \\
\hline & & $0.01 \mathrm{mSv}$ & 987.40 & $\begin{array}{l}80.00 \\
(1.68)\end{array}$ & $\begin{array}{c}\text { Safe area from } \\
8 \mathrm{~km}\end{array}$ \\
\hline
\end{tabular}

During springer, the TEDE obtained in both sites is almost without any significant effect. Whereas, the impact area of TEDE exceeding $0.01 \mathrm{mSv}$ is three times higher in Skhira site than in Marsa Dhouiba site but this finding is not so important because all the green area doesn't represent any danger. 
III.3.2 Thyroid Commitment Dose

(a)

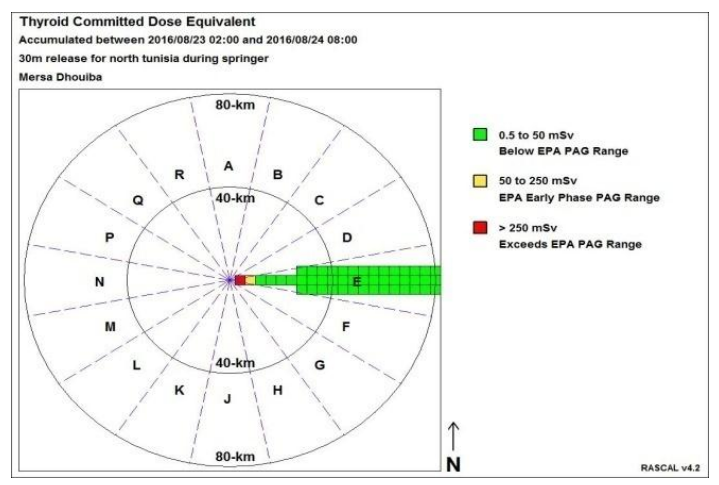

(b)

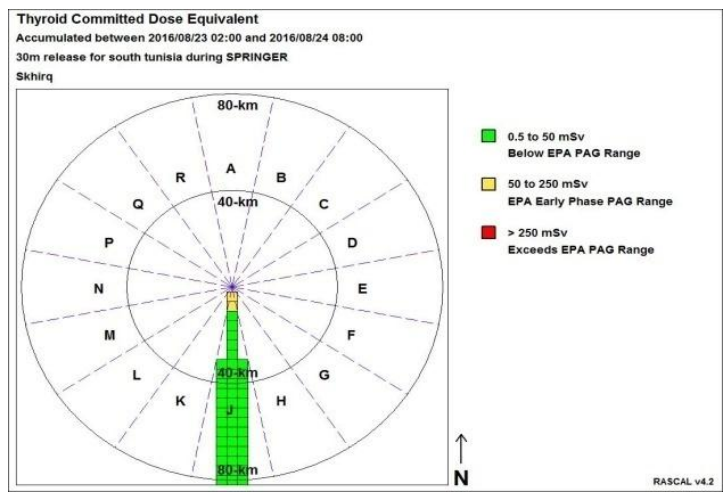

Fig. 7. Schematization Thyroid Commitment Dose obtained during springer season (a) Marsa-Dhouiba site (Met. Stab class, D) (b) Skhira site (Met. Stab class, C)

Table 11. RASCAL's TCDE outputs obtained during autumn for both

\begin{tabular}{|c|c|c|c|c|c|}
\hline \\
\hline Site & $\begin{array}{c}\text { Maximum } \\
\text { dose [Sv] } \\
\text { (Distance } \\
[\mathrm{m}])\end{array}$ & $\begin{array}{c}\text { Threshold } \\
\text { TCDE > }\end{array}$ & $\begin{array}{c}\text { Impact } \\
\text { area } \\
{[\mathrm{km} 2]}\end{array}$ & $\begin{array}{c}\text { Maximum } \\
\text { distance } \\
\text { reached } \\
{[\mathrm{km}]} \\
(\mathrm{values} \\
[\mathrm{mSv}])\end{array}$ & $\begin{array}{l}\text { Recommended } \\
\text { immediate } \\
\text { protective actions }\end{array}$ \\
\hline \multirow{3}{*}{ 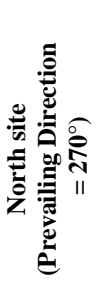 } & \multirow{3}{*}{$\begin{array}{l}5.55 \\
(800)\end{array}$} & $250 \mathrm{mSv}$ & 16.20 & $\begin{array}{c}4.00 \\
(271.00)\end{array}$ & $\begin{array}{l}\text { KI admission for } \\
\text { adults up to } 40 \text { years } \\
\text { for under } 4 \mathrm{~km}\end{array}$ \\
\hline & & $50 \mathrm{mSv}$ & 32.40 & $\begin{array}{c}8.00 \\
(73.90)\end{array}$ & $\begin{array}{c}\text { KI admission for } \\
\text { children, pregnant } \\
\text { and lactating woman } \\
\text { for under } 8 \mathrm{~km}\end{array}$ \\
\hline & & $0.05 \mathrm{mSv}$ & 874.10 & $\begin{array}{l}80.00 \\
(9.12)\end{array}$ & Safe area from $8 \mathrm{~km}$ \\
\hline \multirow{3}{*}{ 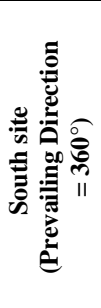 } & \multirow{3}{*}{$\begin{array}{l}4.89 \\
(800)\end{array}$} & $250 \mathrm{mSv}$ & 0.10 & $\begin{array}{c}3.22 \\
(352.00)\end{array}$ & $\begin{array}{l}\mathrm{KI} \text { admission for } \\
\text { adults up to } 40 \text { years } \\
\text { for under } 3.22 \mathrm{~km}\end{array}$ \\
\hline & & $50 \mathrm{mSv}$ & 0.40 & $\begin{array}{c}8.00 \\
(59.90)\end{array}$ & $\begin{array}{c}\text { KI admission for } \\
\text { children, pregnant } \\
\text { and lactating woman } \\
\text { for under } 8 \mathrm{~km}\end{array}$ \\
\hline & & $0.05 \mathrm{mSv}$ & 841.70 & $\begin{array}{l}80.00 \\
(4.98)\end{array}$ & $\begin{array}{c}\text { Safe area from } 8 \\
\mathrm{~km}\end{array}$ \\
\hline
\end{tabular}

\section{III.4. Atmospheric dispersion during summer}

III.4.1 TEDE

(a)

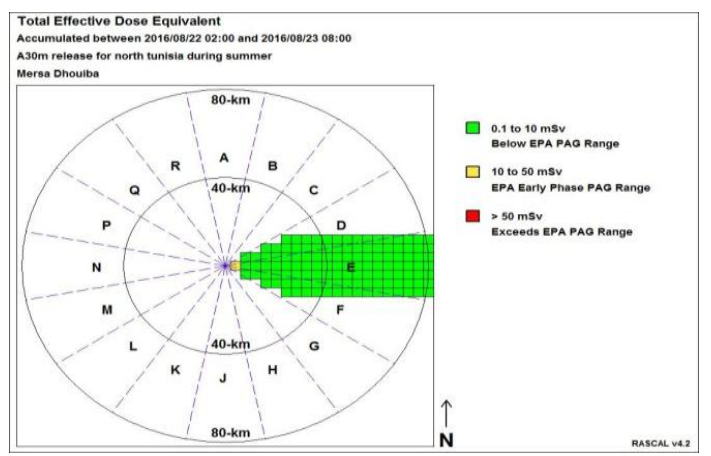

(b)

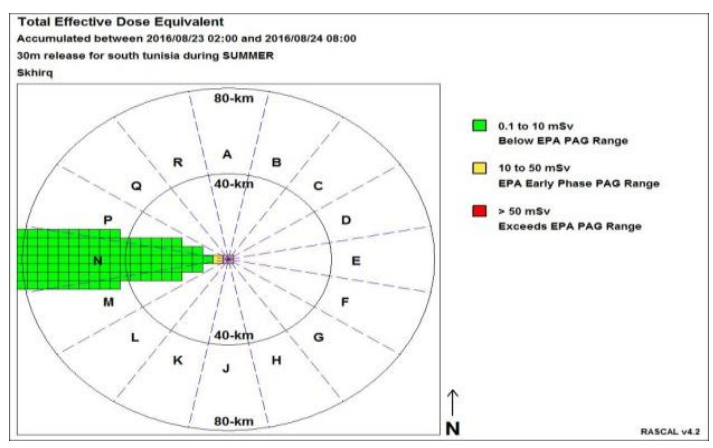

Fig. 8. Schematization of Simulated TEDE obtained during summer season (a) Marsa-Dhouiba site (Met. Stab class, D) (b) Skhira site (Met. Stab class, C)

Table 12. RASCAL's TEDE outputs obtained during summer for both sites

\begin{tabular}{|c|c|c|c|c|c|}
\hline Site & $\begin{array}{c}\text { Maximum } \\
\text { dose }[\mathrm{Sv}] \\
\text { (Distance } \\
[\mathrm{m}])\end{array}$ & $\begin{array}{l}\text { Threshold } \\
\text { TEDE > }\end{array}$ & $\begin{array}{c}\text { Impact } \\
\text { area } \\
{[\mathrm{km} 2]}\end{array}$ & $\begin{array}{c}\text { Maximum } \\
\text { distance } \\
\text { reached } \\
{[\mathrm{km}]} \\
\text { (values } \\
[\mathrm{mSv}])\end{array}$ & $\begin{array}{l}\text { Recommended } \\
\text { immediate } \\
\text { protective } \\
\text { actions }\end{array}$ \\
\hline \multirow{3}{*}{ 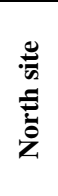 } & \multirow{3}{*}{$\begin{array}{l}0.266 \\
(800)\end{array}$} & $50 \mathrm{mSv}$ & 0.00 & $\begin{array}{c}1.61 \\
(77.60)\end{array}$ & $\begin{array}{c}\text { Evacuation to } \\
1.61 \mathrm{~km}\end{array}$ \\
\hline & & $10 \mathrm{mSv}$ & 0.30 & $\begin{array}{c}3.22 \\
(15.10)\end{array}$ & $\begin{array}{c}\text { Sheltering to } \\
3.22 \mathrm{~km}\end{array}$ \\
\hline & & $0.01 \mathrm{mSv}$ & 2784.2 & $\begin{array}{l}80.00 \\
(0.70)\end{array}$ & $\begin{array}{c}\text { Safe area from } \\
3.22 \mathrm{~km}\end{array}$ \\
\hline \multirow{3}{*}{ 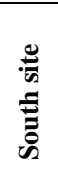 } & \multirow{3}{*}{$\begin{array}{l}0.392 \\
(800)\end{array}$} & $50 \mathrm{mSv}$ & 0.10 & $\begin{array}{c}2.41 \\
(59.1)\end{array}$ & $\begin{array}{c}\text { Evacuation to } \\
2.41 \mathrm{~km}\end{array}$ \\
\hline & & $10 \mathrm{mSv}$ & 0.40 & $\begin{array}{c}4.83 \\
(11.7) \\
\end{array}$ & $\begin{array}{c}\text { Sheltering to } \\
4.83 \mathrm{~km}\end{array}$ \\
\hline & & $0.01 \mathrm{mSv}$ & 2282.4 & $\begin{array}{l}80.00 \\
(0.80)\end{array}$ & $\begin{array}{c}\text { Safe area from } \\
4.83 \mathrm{~km}\end{array}$ \\
\hline
\end{tabular}

Also, in summer, the TEDE obtained in both sites is almost without any significant effect. Whereas, the impact area of TEDE exceeding $0.01 \mathrm{mSv}$ is three times higher in MarsaDhouiba site than in Skhira site but this finding is not so important because all the green area doesn't represent any danger. 
III.4.2 Thyroid Commitment Dose

(a)

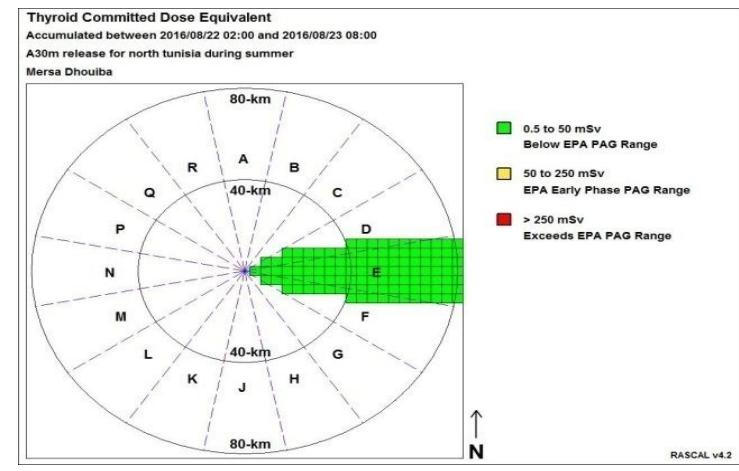

(b)

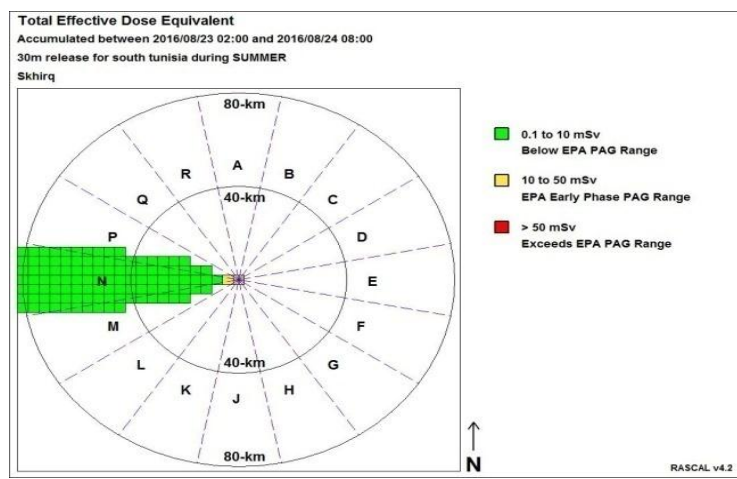

Fig. 9. Schematization Thyroid Commitment Dose obtained during summer season (a) Marsa-Dhouiba site (Met. Stab class, D) (b) Skhira site (Met. Stab class, C)

Table 13. RASCAL's TCDE outputs obtained during autumn for both

\begin{tabular}{|c|c|c|c|c|c|}
\hline Site & $\begin{array}{c}\text { Maximum } \\
\text { dose [Sv] } \\
\text { (Distance } \\
[\mathrm{m}])\end{array}$ & $\begin{array}{c}\text { Threshold } \\
\text { TCDE > }\end{array}$ & $\begin{array}{c}\text { Impact } \\
\text { area } \\
{[\mathrm{km} 2]}\end{array}$ & $\begin{array}{c}\text { Maximum } \\
\text { distance } \\
\text { reached } \\
{[\mathrm{km}]} \\
\text { (values } \\
[\mathrm{mSv}])\end{array}$ & $\begin{array}{l}\text { Recommended } \\
\text { immediate } \\
\text { protective actions }\end{array}$ \\
\hline \multirow{3}{*}{ 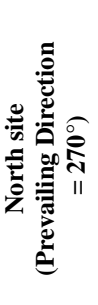 } & \multirow{3}{*}{$\begin{array}{l}0.839 \\
(800)\end{array}$} & $250 \mathrm{mSv}$ & 0.0 & $\begin{array}{c}0.80 \\
(839.00)\end{array}$ & $\begin{array}{l}\text { KI admission for } \\
\text { adults up to } 40 \text { years } \\
\text { for under } 800 \mathrm{~km}\end{array}$ \\
\hline & & $50 \mathrm{mSv}$ & 0.2 & $\begin{array}{c}3.22 \\
(54.20)\end{array}$ & $\begin{array}{l}\text { KI admission for } \\
\text { children, pregnant } \\
\text { and lactating woman } \\
\text { for under } 3.22 \mathrm{~km} \\
\end{array}$ \\
\hline & & $0.05 \mathrm{mSv}$ & 2622.4 & $\begin{array}{l}80.00 \\
(2.25)\end{array}$ & $\begin{array}{c}\text { Safe area from } 3.22 \\
\mathrm{~km}\end{array}$ \\
\hline \multirow{3}{*}{ 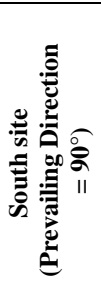 } & \multirow{3}{*}{$\begin{array}{c}1.40 \\
(800)\end{array}$} & $250 \mathrm{mSv}$ & 469.4 & $\begin{array}{c}1.61 \\
(363.00)\end{array}$ & $\begin{array}{l}\text { KI admission for } \\
\text { adults up to } 40 \text { years } \\
\text { for under } 1.61 \mathrm{~km}\end{array}$ \\
\hline & & $50 \mathrm{mSv}$ & 598.9 & $\begin{array}{c}3.22 \\
(92.20)\end{array}$ & $\begin{array}{c}\text { KI admission for } \\
\text { children, pregnant } \\
\text { and lactating woman } \\
\text { for under } 3.22 \mathrm{~km} \\
\end{array}$ \\
\hline & & $0.05 \mathrm{mSv}$ & 825.6 & $\begin{array}{l}80.00 \\
(2.46)\end{array}$ & $\begin{array}{c}\text { Safe area from } 3.22 \\
\mathrm{~km}\end{array}$ \\
\hline
\end{tabular}

\section{III.5. Worst scenario of release}

We are interesting in study the worst possible scenario of release, in terms of meteorological conditions, leading to the highest activity in the air. So, we assume a calm winds whose wind speed is $1 \mathrm{~m} / \mathrm{s}$ and a very stable atmosphere ( $\mathrm{F}$ stability class). The relative humidity is also considered higher (90\%), where these meteorological conditions lead to worst dispersion conditions, (Figure 10 and Table 14).

(a)

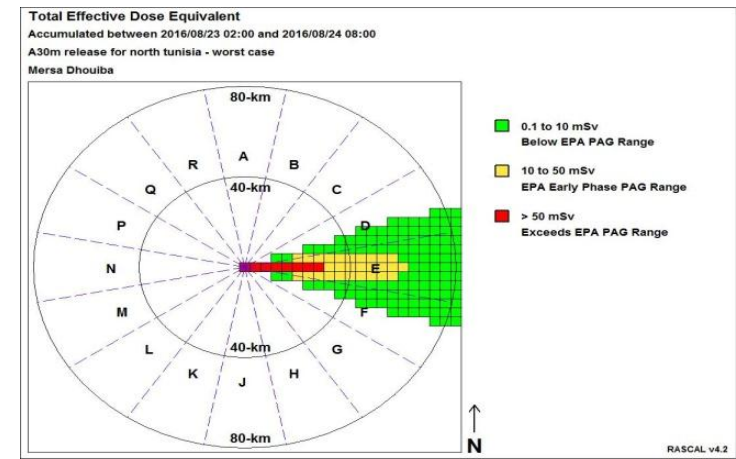

(b)

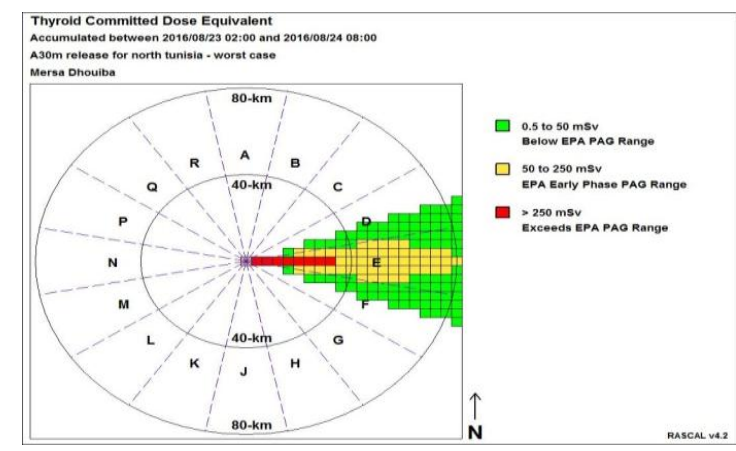

Fig. 10 Schematization of Simulated doses obtained during worst dispersion conditions for (a) TEDE (b) TCDE

Table 14. RASCAL's dose outputs obtained for the worst release case

\begin{tabular}{|c|c|c|c|c|c|}
\hline Dose & Threshold & $\begin{array}{c}\text { Maximum } \\
\text { dose }[\mathrm{Sv}] \\
\text { (Distance } \\
[\mathrm{m}])\end{array}$ & $\begin{array}{c}\text { Impact } \\
\text { area } \\
{[\mathrm{km} 2]}\end{array}$ & $\begin{array}{c}\text { Maximum } \\
\text { distance } \\
\text { reached } \\
{[\mathbf{k m}]} \\
\text { (values } \\
[\mathrm{mSv}])\end{array}$ & $\begin{array}{l}\text { Recommended } \\
\text { immediate } \\
\text { protective } \\
\text { actions }\end{array}$ \\
\hline \multirow{3}{*}{$\hat{\hat{\mathbf{x}}}$} & $50 \mathrm{mSv}$ & \multirow{3}{*}{$\begin{array}{l}2.60 \\
(800)\end{array}$} & 978.40 & $\begin{array}{c}28 \\
(52.80)\end{array}$ & $\begin{array}{c}\text { Evacuation to } \\
28 \mathrm{~km}\end{array}$ \\
\hline & $10 \mathrm{mSv}$ & & 1796.80 & $\begin{array}{c}60 \\
(10.80)\end{array}$ & $\begin{array}{l}\text { Sheltering to } \\
60 \mathrm{~km}\end{array}$ \\
\hline & $0.01 \mathrm{mSv}$ & & 2638.60 & $\begin{array}{l}80.00 \\
(5.37)\end{array}$ & $\begin{array}{c}\text { Safe area from } \\
60 \mathrm{~km}\end{array}$ \\
\hline \multirow{3}{*}{$\hat{\text { 됭 }}$} & $250 \mathrm{mSv}$ & \multirow{3}{*}{$\begin{array}{l}11.10 \\
(800)\end{array}$} & 48.60 & $\begin{array}{c}8.00 \\
(336)\end{array}$ & $\begin{array}{c}8 \mathrm{~km}(336 \\
\mathrm{mSv})\end{array}$ \\
\hline & $10 \mathrm{mSv}$ & & 129.50 & $\begin{array}{c}28 \\
(52.80)\end{array}$ & $\begin{array}{c}28 \mathrm{~km}(52.8 \\
\mathrm{mSv})\end{array}$ \\
\hline & $0.01 \mathrm{mSv}$ & & 2573.60 & $\begin{array}{l}80.00 \\
(5.37)\end{array}$ & $\begin{array}{c}80 \mathrm{~km}(5.37 \\
\mathrm{msv})\end{array}$ \\
\hline
\end{tabular}




\section{III.6. Worst scenario of release}

Table 15 gives the extension of area where early protection actions must be taken. It is found that most extending areas are obtained for northern site for winter, autumn and Springer seasons except during summer when highest areas are given for South site. Also, we notice that highest seasonal evacuation and sheltering areas are obtained under meteorological conditions of winter with a maximum evacuation and sheltering areas in Marsa-Dhouiba site, respectively, $11 \mathrm{~km}$ and $20 \mathrm{~km}$. Whereas, if we consider the worst release scenario (calm wind, very stable atmosphere and higher relative humidity), the maximum evacuation and sheltering zones are respectively $28 \mathrm{~km}$ and $60 \mathrm{~km}$. This is the worst possible case we may have.

Table 15. Early protection actions for hyptotetical accident for each season and for worst release case

\begin{tabular}{|c|c|c|c|c|}
\hline Season & site & $\begin{array}{c}\text { Max } \\
\text { Evacuatio } \\
\mathbf{n} \text { area } \\
{[\mathbf{k m}]}\end{array}$ & $\begin{array}{c}\text { Max } \\
\text { Sheltering } \\
\text { area }[\mathbf{k m}]\end{array}$ & $\begin{array}{c}\text { KI } \\
\text { admission }\end{array}$ \\
\hline \multirow{2}{*}{ Winter } & North & 11.27 & 20 & 8.4 \\
\cline { 2 - 5 } & South & 8 & 16 & 3.22 \\
\hline Autumn & North & 4.83 & 8 & 8.04 \\
\cline { 2 - 5 } & South & 2.41 & 4.48 & 8.4 \\
\hline Springer & North & 4.8 & 20 & 8 \\
\cline { 2 - 5 } & South & 4.83 & 8 & 8 \\
\hline Summer & North & 1.61 & 3.22 & 3.22 \\
\cline { 2 - 5 } & South & 2.41 & 4.83 & 3.22 \\
\hline $\begin{array}{c}\text { Worst } \\
\text { Case }\end{array}$ & Both site & 28 & 60 & 28 \\
\hline
\end{tabular}

It is clear that for both sites, the evacuation and sheltering areas are not very wide this is explained by the fact that the two preselected sites are characterized mainly by a neutral and unstable meteorological stability (Table 5).

\section{CONCLUSION}

For accidental atmospheric dispersion following the hypothetical accident of Loss of Coolant Accident, defining as uncovered core events accident, was been treated using RASCAL code. Two mainly results are obtained: the Total Effective Dose Equivalent (TEDE) and the Thyroid Commitment Dose Equivalent (TCDE) around the reactor sites. According to dose's thresholds for TEDE and TCDE, we highlight three main areas extensions colored in green (safety area), yellow (intermediate zone) and red (hazardous area). A protection measures shall be applied in the short distance (approximately less than $5 \mathrm{~km}$ ) due to the good atmospheric parameters but this distance extends for much distances in case of northern site. RASCAL simulations were done for both sites and for every season taking into account its specific meteorological conditions. It was been demonstrated that under meteorological conditions of winter season, relatively we obtained the highest doses and the broadest extensions for 2 sites. For instance, in winter, if we consider the EPA limits, for the northern site, the evacuation zone on within that TEDE exceeds $50 \mathrm{mSv}$ reached up to $11 \mathrm{~km}$ for north site, the sheltering zone (TEDE>10 mSv) is estimated at $20 \mathrm{~km}$ and the admission of KI is recommended within $8 \mathrm{~km}$.

We have also simulated the worst release scenario assuming a calm winds (wind speed is $1 \mathrm{~m} / \mathrm{s}$ ), a very stable atmosphere ( $\mathrm{F}$ stability class) and the highest relative humidity $(\mathrm{HR}=90 \%)$ and it is found that the maximum evacuation and sheltering zones are respectively $28 \mathrm{~km}$ and $60 \mathrm{~km}$. Not that and as a result of the analysis the meteorological conditions of the two sites, we find that these conditions rarely occur in two sites.

Finally, we can conclude that the atmospheric dispersion of the hypothetical accident under specific meteorological stabilities, which are almost neutral and unstable, of the two preselected sites, has no significant health effects because either evacuation or sheltering areas are not very extended and the highest seasonal evacuation and sheltering areas are obtained under meteorological conditions of winter with a maximum evacuation and sheltering areas in Marsa-Dhouiba site, respectively, $11 \mathrm{~km}$ and $20 \mathrm{~km}$. Whereas, these areas could reached $28 \mathrm{Km}$ for evacuation and $60 \mathrm{~km}$ for sheltering if the accident occurs during the worst meteorological conditions.

\section{ACKNOWLEDGMENTS}

The authors highly acknowledge the financial and technical support provided by the IAEA and express their appreciations to the PMO and the PO for their strong commitments and guidance for conducting this work. Also, the authors wish to thank the Division of Africa in the IAEA for their assistance. This work was preformed within the framework of the IAEA regional project RAF/ 7/009.

\section{REFERENCE}

[1] Bamdad H., and John M. (2016). Bayesian estimation of airborne fugitive emissions using a Gaussian plume model. Atmospheric Environment. Vol. 141, pp. 122-138.

[2] Benamrane Y., and Boustras G. (2015). Atmospheric dispersion and impact modeling system:how are they perceived as support tools for nuclear crises management. .Safety Science Vol. 71, pp. 48-55

[3] Benamrane Y., Wybo, J., and Armand, P. (2013). Cherbobyl and Fukushima nuclear accidents: what has changed in the use of atmospheric dispersion modeling?.Journal of Environmental Radioactivity. Vol. 126, pp. 239-252

[4] Bo C., Junxiao Z., and Yixue C. (2016). Radiation Dose Calculations for a Hypothetical Accident in Xianning Nuclear Power Plant; Hindawi Publishing Corporation 
Science and Technology of Nuclear Installations, Vol. 2016, Article ID 3105878, 6 pages.

[5] Denglong M. and Zaoxiao Z. (2016). Contaminant dispersion prediction and source estimation with integrated Gaussian-machine learning network model for point source emission in atmosphere. Journal of Hazardous Materials. Vol. 311, pp. 237-245

[6] http://www.nrc.gov/reactors/new-reactors/esp.html

[7] McGuirea S.A., J.V. Ramsdell, Jr.B., and G.F. Atheyc. (2007); RASCAL 3.0.5: Description of Models and Methods. U.S. Nuclear Regulatory Commission Office of Nuclear Security and Incident Response Washington, DC 20555-0001

[8] Muswema J., Darko E., Gbadago J. and Boafo E. (2014). Atmospheric dispersion modeling and radiological safety analysis for a hypothetical accident of Ghana Research Reactor-1 (GHARR-1). Annals of Nuclear Energy, Vol. 68, pp. 239-246

[9] Pirouzmand A., Dehghani P., Hadad K. and Nematollahi M. (2015). Dose assessment of radionuclides dispersion from Bushehr nuclear power plant stack under normal operation and accident Conditions. International Journal of Hydrogen Energy, Vol. 40, No. 44, pp. 15198-15205

[10] Rossi F., Guglielmelli A. and Rocchi F. (2014). Impact of a security event at a TRIGA reactor. Annals of Nuclear Energy Vol. 76 pp. 125-136.

[11] Schmidt H. and Ivanov I. (2012). Environmental Consequences and Management of a Severe Accident, Chapter 7 in Nuclear Safety in Light Water Reactors, pp. Academic Press, 1 edition, ISBN-10: 0123884462, pp 589-624.

[12] Soffer L., Burson B., Ferrell C., Lee, Y. and Ridgely J. N. (1995). Accident Source Terms for Light-Water Nuclear Power Plants, NUREG-1465.

[13] Timothy J. (2013). Comparison of chlorine and ammonia concentration field trial data with calculated results from a Gaussian atmospheric transport and dispersion model. Original Research Article Journal of Hazardous Materials, Vol. 254-255, pp. 325-335.

[14] U.S. Environmental Protection Agency (2013). Protective Action Guides and Planning Guidance For Radiological Incidents, Draft for Interim Use and Public Comment (March 2013).

[15] U.S. Food and Drug Administration (FDA), (2001, 2002). U.S. Department of Health and Human Services, Food and Drug Administration (FDA) Center for Drug Evaluation and Research (CDER).. 